\title{
Dispositivos de tecnologia assistiva: fatores relacionados ao abandono ${ }^{1}$
}

\author{
Celso Rodrigues da Costa ${ }^{a}$, Fernanda Márcia Rodrigues Martins Ferreira ${ }^{a}$, \\ Marcos Vinícius Bortolus ${ }^{\mathrm{b}}$, Maria Gabriela Reis Carvalho ${ }^{\mathrm{a}}$ \\ aUniversidade Federal de Minas Gerais - UFMG, Belo Horizonte, MG, Brasil. \\ ${ }^{b}$ Departamento de Engenharia Mecânica, Universidade Federal de Minas Gerais - UFMG, \\ Belo Horizonte, MG, Brasil.
}

\begin{abstract}
Resumo: Em face do elevado número de indivíduos com deficiências, bem como da importância dos dispositivos de tecnologia assistiva (DTAs) para a melhoria de seu desempenho ocupacional, as elevadas taxas de abandono destes dispositivos e os altos custos a estes associados, esta revisão tem o objetivo de identificar fatores relacionados ao abandono de dispositivos de tecnologia assistiva. A metodologia utilizada foi uma revisão crítica da literatura utilizando as palavras-chave: tecnologia assistiva/assistive technology, abandono/abandonment, rejeição/rejection, e desuso/disuse, de publicações entre os anos de 2002 e 2013. Foi realizada a busca nas bases de dados MEDLINE, LILACS e SciELO, e identificado um total de 49 artigos, sendo que nove publicações atenderam aos critérios de inclusão. Constatou-se que os fatores mais citados relacionados ao abandono dos dispositivos são: problemas com o estado físico do usuário; falta de informação e treinamento tanto de profissionais quanto de usuários; dor; limitações funcionais; preferência por outro DTA ou utilização de capacidades remanescentes; peso elevado; alterações nas condições do DTA; dificuldade de uso; insatisfação; desconforto; inadequação/inapropriação, e "muito barulho". Compreender melhor esses fatores é fundamental para melhorar a eficácia da prescrição e da intervenção por parte dos profissionais, o que é imprescindível para aumentar a adesão e promover resultados efetivos.
\end{abstract}

Palavras-chave: Tecnologia Assistiva, Rejeição, Dispositivos, Ação Terapêutica, Cadeiras de Rodas, Sistemas de Comunicação Alternativos e Aumentativos.

\section{Assistive technology devices: abandonment related factors}

\begin{abstract}
This review aims to identify factors related to abandonment of assistive technology devices in face of the increased number of individuals with disabilities, as well as the importance of the assistive technology devices (ATD) to improve their occupational performance, the increased abandonment rates of these devices and the high costs associated with them. We used a critical literature review with the keywords: assistive technology/tecnologia assistiva, abandonment/abandono, rejection/rejeição, and disuse/desuso, published between the years 2002 and 2013. The search was conducted in MEDLINE, LILACS and SciELO and identified a total of 49 articles and 9 publications that met the inclusion criterion. It found that the most cited factors related to the abandonment of devices are problems with the user's physical condition; lack of information and training both professionals and users; pain; lack of functionality; ATD preference or other use of remaining capacity; increased weight; changes in ADT conditions; use difficulty; unsatisfactoriness; discomfort; inadequacy/inappropriate; and "lot of noise". The better understanding of these factors is critical to improve the effectiveness of prescription and intervention by professionals and imperative to increase membership and promote effective results.
\end{abstract}

Keywords: Assistive Technology, Rejection, Devices, Therapeutics, Wheelchairs, Augmentative and Alternative Communications Systems.

Autor para correspondência: Celso Rodrigues da Costa, Ortopédica LTDA, Rua Desembargador Bráulio, 3047, Taquaril, CEP 30290-020, Belo Horizonte, MG, Brasil, e-mail: tocelso@hotmail.com

Recebido em Ago. 27, 2014; $1^{\text {a }}$ Revisão em Jan.19, 2015; 2 ${ }^{\text {a }}$ Revisão em Mar. 24, 2015; Aceito em Abr. 7, 2015. 


\section{Introdução}

Segundo a Organização Mundial de Saúde (ORGANIZAÇÃO..., 2012), em todo o mundo, mais de um bilhão de pessoas possui alguma deficiência, sendo que cerca de 200 milhóes experimentam dificuldades funcionais consideráveis. A OMS afirma ainda que quase todas as pessoas terão uma deficiência, seja temporária ou permanente, em algum momento de suas vidas, e enfrentarão dificuldades cada vez maiores com a funcionalidade de seus corpos, por causa do envelhecimento. Apenas no Brasil, de acordo com os resultados do Censo Demográfico de 2010, disponibilizados pelo Instituto Brasileiro de Geografia e Estatística, a populaçáo que se declara com algum tipo de deficiência se elevou de $14,5 \%$ (INSTITUTO..., 2003) para 23,9\% (INSTITUTO..., 2012). Esses valores indicam um crescimento de $65 \%$ em dez anos. A Classificação Internacional de Funcionalidade, Incapacidade e Saúde (CIF), elaborada pela OMS, conceitua deficiência como "[...] problemas nas funções ou nas estruturas do corpo, tais como um desvio importante ou uma perda [...]" (ORGANIZAÇÃO..., 2003, p.13).

As pessoas com deficiência comumente apresentam dificuldades e precisam de auxílio para conseguir realizar atividades funcionais no seu dia a dia (OLIVEIRA; GAROTTI; SÁ, 2008; BERQUÓ, 2008; ANDRADE; PEREIRA, 2009; CRUZ, 2012). Para ajudá-las a ter melhor desempenho e satisfação em suas atividades, podem ser utilizados dispositivos de tecnologia assistiva (DTAs), definidos no Assistive Technology Act of 1998 (UNITES STATES, 1998, p. 3631, tradução nossa) como "[...] qualquer item, parte de um equipamento ou sistema de produto, adquirido comercialmente, modificado ou personalizado, que é usado para aumentar, manter ou melhorar as capacidades funcionais das pessoas com deficiência".

O DTA tem como objetivo proporcionar independência e inclusão para uma melhor qualidade de vida desta população, diminuindo o impacto dos prejuízos físicos, cognitivos e sociais, melhorando o desempenho funcional da pessoa (HOGETOP; SANTAROSA, 2002). Dessa forma, o DTA faz parte do campo da tecnologia assistiva (TA). No Brasil, o Comitê de Ajudas Técnicas aprovou a adoção do conceito de TA como:

Uma área do conhecimento, de característica interdisciplinar, que engloba produtos, recursos, metodologias, estratégias, práticas e serviços que objetivam promover a funcionalidade, relacionada à atividade e participação de pessoas com deficiência, incapacidades ou mobilidade reduzida, visando a autonomia, independência, qualidade de vida e inclusão social (BRASIL, 2007, p.3).

Segundo Mello (1999), a tecnologia é considerada "assistiva" quando é utilizada para auxiliar o indivíduo a desempenhar uma atividade funcional e pode ser classificada em: simples ou sofisticada; concreta ou teórica; equipamento (não necessita de treinamento $\mathrm{e}$ habilidades) ou instrumento (depende de habilidades específicas para a utilização); geral (utilizada em diversas atividades) ou específica (para determinada atividade); comercializada (atende grande parte das pessoas com incapacidade) ou individualizada (feita sob medida). A autora ainda pontua as principais áreas de aplicaçấo da TA, que são: auxílios para a vida diária e prática; comunicação alternativa; recursos de acessibilidade ao computador (informática); sistemas de controle de ambiente; projetos arquitetônicos para acessibilidade; órteses e próteses; adequação postural; mobilidade; auxílios para cegos ou com visão subnormal; auxílios para surdos ou com déficit auditivo, e adaptações em veículos.

A equipe responsável pela seleção, pela prescriçãao e pelo uso de dispositivos pode ser composta por diversos profissionais, como Fisioterapeutas, Fonoaudiólogos, Musicoterapeutas, Médicos, Advogados, Arquitetos e Engenheiros, contribuindo com conhecimento e habilidades específicas de sua área. Normalmente, o Terapeuta Ocupacional é o coordenador de um processo de prescriçáo de TA e, para isso, deve ter profundo conhecimento na aplicação da tecnologia para suprir as necessidades dos indivíduos, sendo necessário também manter-se atualizado em relação aos produtos disponíveis no mercado, pois a cada ano novos produtos são lançados (MELLO, 1999).

Um grande problema no campo da TA é o abandono dos dispositivos. Estudos mostram que cerca de 30\% de todos os dispositivos adquiridos são abandonados pelo usuário entre o primeiro e o quinto ano de uso, e alguns nem mesmo chegam a ser utilizados (PHILLIPS; ZHAO, 1993; RIEMER-REISS; WACHER, 2000). Este abandono tem um impacto negativo em termos econômicos para o indivíduo e para o sistema nacional de saúde.

O Plano Nacional dos Direitos da Pessoa com Deficiência - Viver sem Limite, por meio do Decreto 7.612, de 17 de novembro de 2011, previu um investimento total no valor de 7,6 bilhôes de reais até 2014. Uma das ações deste plano foi o Financiamento para a Aquisição de Bens e Serviços de Tecnologia Assistiva, uma linha de crédito que possibilita a aquisiçẫo de bens e serviços de tecnologia assistiva a pessoas com deficiência (BRASIL, 2013). 
Nos Estados Unidos, cerca de 50\% dos DTAs são parcialmente pagos por terceiros, como instituiçôes de caridade ou subsídios do governo; e, no Canadá, estuda-se o poder potencial de voluntários técnicos em colaboraçáo com o setor de educaçáo para resolver o problema dos altos custos (LI; SELLERS, 2009). Percebe-se que os valores destinados à aquisição de DTA são muito altos e, além de causar prejuízo econômico, o abandono do DTA supóe que as necessidades do usuário não foram supridas. Isto pode ser visto como um fracasso para a equipe, bem como ter um impacto negativo sobre a relação usuário/equipe de saúde.

Considerando-se o elevado número de indivíduos com deficiências, bem como a importância dos DTAs para a melhoria de seu desempenho ocupacional, as elevadas taxas de abandono destes dispositivos e os altos custos a estes associados, faz-se necessário o presente estudo, com o objetivo de identificar e discutir fatores relacionados ao abandono de DTA.

\section{Metodologia}

O estudo utiliza como método a revisão crítica da literatura, que reúne, sintetiza e analisa informaçôes disponibilizadas na literatura, mas não segue necessariamente uma metodologia pré-definida (MANCINI; SAMPAIO, 2006). A pergunta norteadora consistiu em: quais os fatores relacionados ao abandono do dispositivo de tecnologia assistiva?

A seleçáo dos artigos foi feita por meio das bases de dados: MEDLINE (Medical Literature Analysis and Retrieval System on-line), LILACS (Literatura Latino-Americana e do Caribe em Ciências da Saúde) e SciELO (Scientific Electronic Library Online). As buscas foram realizadas por dois autores, utilizando-se as palavras-chave: tecnologia assistiva/assistive technology, abandono/abandonment, rejeição/rejection e desuso/disuse. Foi feita combinação variada entre as palavras-chave para ampliar/limitar a busca, com obrigatoriedade do descritor controlado do DeCS (Descritores em Ciências da Saúde) tecnologia assistiva/assistive technology.

Os critérios de inclusão de artigos foram: abordar o uso de tecnologia assistiva, apontando fatores ligados ao abandono ou à rejeição de DTA; estar publicado em inglês ou português, e ter sido publicado entre 2002 e 2013. Foram excluídos os artigos que não abordavam as razóes do abandono ou da rejeição dos DTAs. Os artigos que atingiram os critérios de inclusão foram analisados criticamente, utilizando a metodologia proposta por Law et al. (1998) como instrumento norteador, a fim de se analisarem de forma estruturada e clara os objetivos, justificativas, desenhos dos estudos, características da amostra e protocolos, resultados, conclusôes e implicações clínicas.

\section{Resultados}

$\mathrm{Na}$ revisão crítica da literatura, foi identificado um total de 49 artigos, dos quais 11 foram pré-selecionados pelo conteúdo do título e do resumo. Destes, dois foram excluídos após a leitura, por não apresentarem fatores ligados ao abandono dos DTAs utilizados. Assim, para o presente estudo, foram selecionados nove artigos, sendo estes resumidos de forma padronizada, com base nos seguintes tópicos: autor(es) e ano de publicação; objetivo do estudo; caracterização da amostra; protocolo de coleta de dados (Tabela 1); principais resultados, e fatores relacionados ao abandono dos DTAs (Tabela 2).

A partir da análise dos artigos, verifica-se que estes apresentam variações referentes ao tamanho da amostra (variou desde três até 275 indivíduos), idade (variou de 18 a 94 anos), condiçôes clínicas, tempo de uso e tipos do DTA. A idade e os tipos de dispositivos são semelhantes em três artigos (LAFERRIER et al., 2010; GAILEY et al., 2010; MCFARLAND et al., 2010), que fazem parte da mesma edição de uma revista e possuíam a mesma justificativa de estudo, a de explorar o efeito da mudança de paradigma de reabilitação, comparando veteranos da Guerra do Vietná com veteranos da Guerra do Iraque e do Afeganistão. A Figura 1 apresenta a quantidade de artigos que abordam os determinados tipos de DTA abandonados.

De acordo com a forma de coleta de dados, três artigos utilizaram as entrevistas semiestruturadas (KITTEL; MARCO; STEWART, 2002; SMITH et al., 2002; FAGER et al., 2006) e seis artigos utilizaram a entrevista estruturada (VERZA et al., 2006; LAFERRIER et al., 2010; GAILEY et al., 2010; MCFARLAND et al., 2010; MUKHERJEE; SAMANTA, 2005; JOHNSON et al., 2006). Em dois artigos, foram utilizadas entrevistas com grupos focais (SMITH et al., 2002; FAGER et al., 2006).

Dos nove artigos incluídos, sete utilizaram metodologia quantitativa e dois utilizaram metodologia qualitativa, com o intuito de investigar a percepção dos usuários de suas necessidades relacionadas com os DTAs e o impacto destes em suas vidas. Um artigo foi classificado como estudo quase experimental (VERZA et al., 2006) e os demais como estudos transversais. 
Os artigos que compóem esta revisão apresentam 29 diferentes fatores ligados ao abandono de DTA. Foi feita uma relação entre eles para agrupar os que tinham estreita ligação. A Figura 2 mostra os fatores que se repetiram duas ou mais vezes.

\section{Discussão}

Este estudo teve como objetivo identificar fatores relacionados ao abandono de DTA; no entanto, também incluímos os fatores associados à rejeição, ou seja, quando o DTA náo é aceito ou utilizado. Nota-se que foram utilizados diferentes conceitos para abordar a rejeição, como o abandono do equipamento antes de ser utilizado, e outros autores referiram outros fatores, como a interrupção após o uso (VERZA et al., 2006; GAILEY et al., 2010; MCFARLAND et al., 2010). Os únicos estudos que consideram rejeição como não aceitação do dispositivo foram o artigo de Fager et al. (2006), que relata sobre dois indivíduos que optaram por rejeitar o dispositivo de CAA de alta tecnologia:

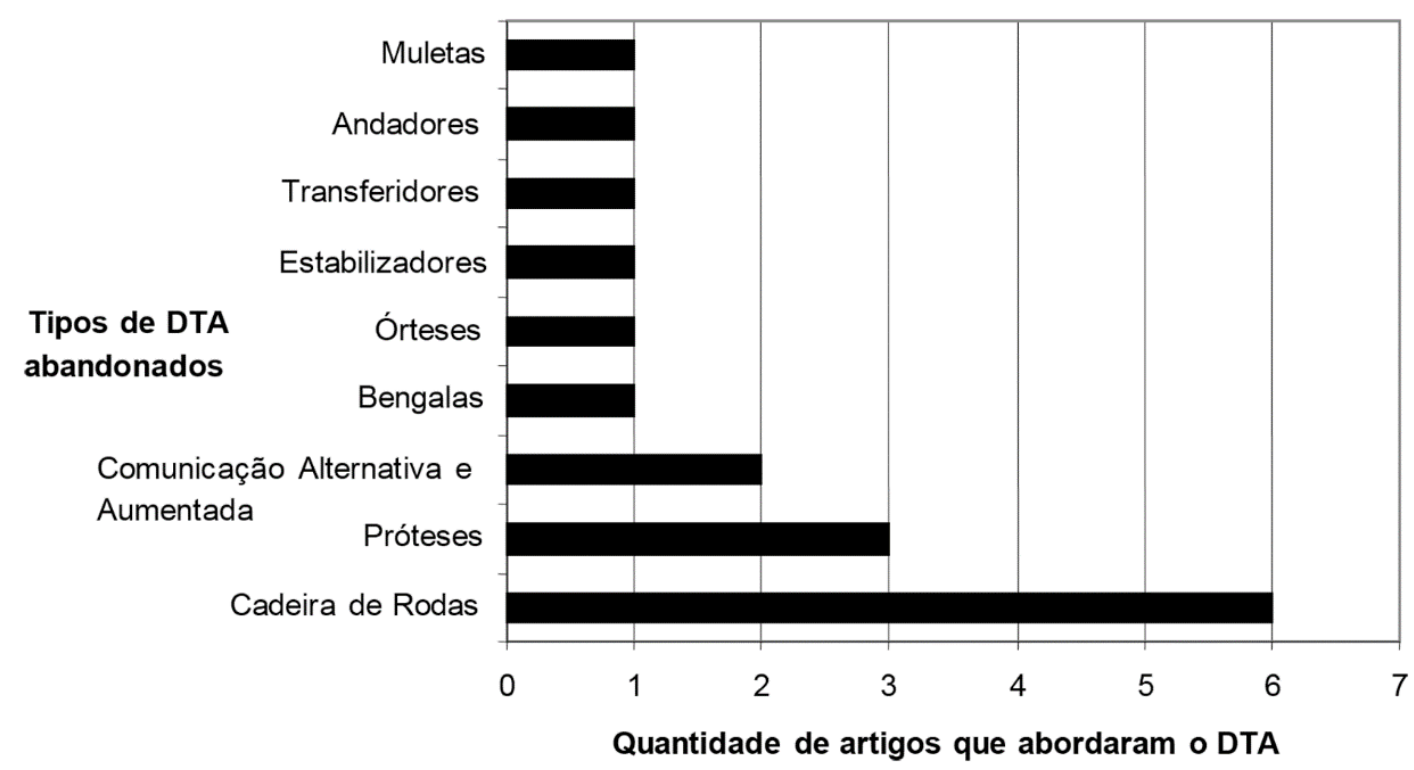

Figura 1. Quantidade de artigos que abordam os determinados tipos de DTA abandonados.

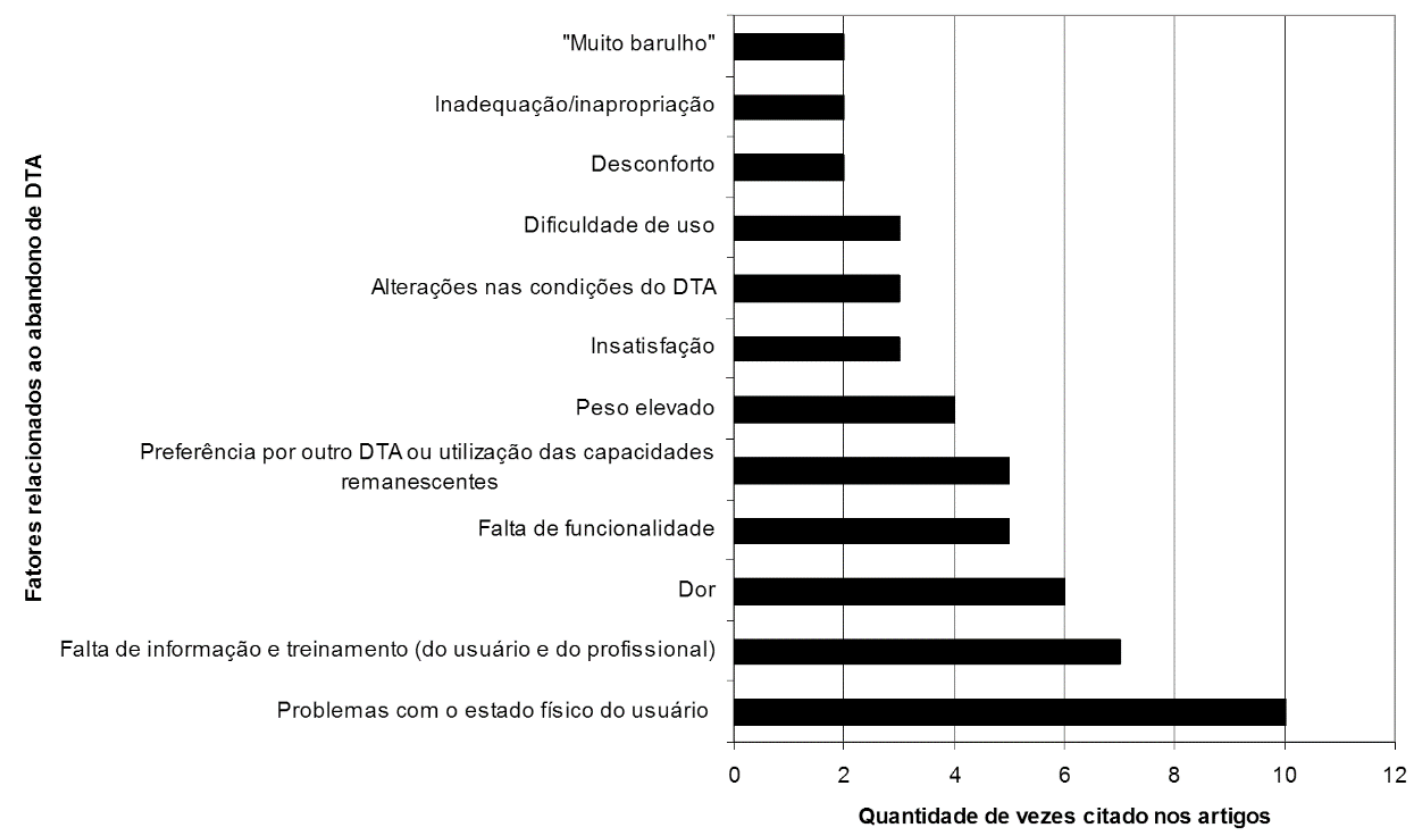

Figura 2. Fatores de abandono de DTA que foram citados duas ou mais vezes. 
Tabela 1. Objetivo, amostra e protocolo de coleta de dados.

\begin{tabular}{|c|c|c|c|}
\hline $\begin{array}{l}\text { Autor e ano de } \\
\text { publicação }\end{array}$ & Objetivo & Amostra & Protocolo de coleta de dados \\
\hline Verza et al. (2006) & $\begin{array}{l}\text { Determinar se } \\
\text { uma abordagem } \\
\text { interdisciplinar } \\
\text { para a avaliação e } \\
\text { a intervenção dos } \\
\text { pacientes que necessitam } \\
\text { de tecnologias assistivas } \\
\text { reduziria o abandono do } \\
\text { dispositivo. } \\
\text { Avaliar os tipos } \\
\text { de dispositivos de } \\
\text { assistência abandonados } \\
\text { por pessoas com } \\
\text { esclerose múltipla e as } \\
\text { razões por que eles não } \\
\text { usaram os dispositivos } \\
\text { que tinham obtido. }\end{array}$ & $\begin{array}{l}\text { Tamanho: } 54 \\
\text { pessoas. } \\
\text { Idade: } 34-70 \text { (pré- } \\
\text { intervenção) e 38-60 } \\
\text { anos (intervenção). } \\
\text { Condições clínicas: } \\
\text { esclerose múltipla. }\end{array}$ & $\begin{array}{l}\text { Os dispositivos foram divididos em: } \\
\text { pré-intervenção (de } 1997 \text { a } 1999 \\
\text { e prescritos pelo fisioterapeuta) e } \\
\text { de intervenção (de } 2000 \text { a } 2002 \mathrm{e} \\
\text { prescritos por equipe composta por } \\
\text { fisioterapeuta, terapeuta ocupacional, } \\
\text { fisiatra e psicólogo). } \\
\text { Os dados do período pré-intervenção } \\
\text { foram coletados a partir dos } \\
\text { prontuários, da documentação pessoal } \\
\text { dos indivíduos (recibos de compras) } \\
\text { e por uma entrevista realizada pelo } \\
\text { fisioterapeuta. Os dados do período } \\
\text { de intervenção foram coletados a } \\
\text { partir do banco de dados de tecnologia } \\
\text { assistiva. }\end{array}$ \\
\hline $\begin{array}{l}\text { Laferrier et al. } \\
\text { (2010) }\end{array}$ & $\begin{array}{l}\text { Determinar fatores } \\
\text { para uso e abandono } \\
\text { de tecnologia assistiva } \\
\text { de mobilidade em } \\
\text { veteranos da guerra do } \\
\text { Vietnã e membros do } \\
\text { serviço e veteranos da } \\
\text { Operação de Liberdade } \\
\text { no Iraque e da Operação } \\
\text { de Liberdade Duradoura } \\
\text { (OIF/OEF), com pelo } \\
\text { menos uma grande perda } \\
\text { traumática dos membros } \\
\text { inferiores. }\end{array}$ & $\begin{array}{l}\text { Tamanho: } 245 \\
\text { veteranos da guerra } \\
\text { do Vietnã e } 226 \\
\text { membros do serviço } \\
\text { e veteranos da OIF/ } \\
\text { OEF. } \\
\text { Idade: } 60,7 \pm 2,9 \\
\text { (Vietnã) e } 29 \pm 5,6 \\
\text { anos (OIF/OEF). } \\
\text { Condições clínicas: } \\
\text { perda unilateral e } \\
\text { bilateral dos membros } \\
\text { inferiores, e com } \\
\text { perda de vários } \\
\text { membros. }\end{array}$ & $\begin{array}{l}\text { Foram adaptadas questões da } \\
\text { Houghton Scale, Orthotic and } \\
\text { Prosthetic User's Survey (OPUS) e } \\
\text { Prosthetic Evaluation Questionnaire } \\
\text { (PEQ). } \\
\text { As perguntas eram respondidas } \\
\text { por telefone, carta ou pela internet } \\
\text { (REIBER et al., 2010). }\end{array}$ \\
\hline $\begin{array}{l}\text { Gailey et al. } \\
(2010)\end{array}$ & $\begin{array}{l}\text { Determinar o uso } \\
\text { e a satisfação de } \\
\text { várias categorias de } \\
\text { dispositivos protéticos e } \\
\text { descrever as capacidades } \\
\text { funcionais de veteranos } \\
\text { e membros do serviço } \\
\text { do Vietnã e da OIF/OEF } \\
\text { com perda unilateral de } \\
\text { membros inferiores. }\end{array}$ & $\begin{array}{l}\text { Tamanho: } 178 \\
\text { veteranos do Vietnã e } \\
\text { 172 das OIF/OEF. } \\
\text { Idade: } \\
60,8 \pm 3,2 \text { (Vietnã) e } \\
29,4 \pm 6,1 \text { anos (OIF/ } \\
\text { OEF). } \\
\text { Condições clínicas: } \\
\text { perda unilateral do } \\
\text { membro inferior. }\end{array}$ & $\begin{array}{l}\text { Foram coletadas informações } \\
\text { sobre a prótese atual e a utilização } \\
\text { de dispositivos (número, tipo e } \\
\text { frequência diária de uso), bem como } \\
\text { a satisfação com próteses e serviços } \\
\text { atuais. Para as próteses que foram } \\
\text { abandonadas devido à insatisfação, } \\
\text { foram coletados dados acerca do } \\
\text { número de tipos de dispositivos e das } \\
\text { razões do abandono. A capacidade } \\
\text { funcional dos membros inferiores } \\
\text { foi avaliada de } 1 \text { (não pode andar, } \\
\text { precisa de ajuda para transferir) até } \\
7 \text { (faz atividades de alto impacto, } \\
\text { como basquete ou esquiar). O estado } \\
\text { de saúde foi autoavaliado e os } \\
\text { participantes forneceram informações } \\
\text { sobre comorbidades, dor e lesões de } \\
\text { combate associadas. } \\
\text { As perguntas eram respondidas por } \\
\text { telefone, carta ou pela internet. }\end{array}$ \\
\hline
\end{tabular}


Tabela 1. Continuação...

\begin{tabular}{|c|c|c|c|}
\hline $\begin{array}{l}\text { Autor e ano de } \\
\text { publicação }\end{array}$ & Objetivo & Amostra & Protocolo de coleta de dados \\
\hline $\begin{array}{l}\text { McFarland et al. } \\
\text { (2010) }\end{array}$ & $\begin{array}{l}\text { Descrever os padrões } \\
\text { de uso dos dispositivos } \\
\text { protéticos em dois } \\
\text { grandes grupos de } \\
\text { membros do serviço e } \\
\text { veteranos, com perda } \\
\text { dos membros superiores } \\
\text { em combate. }\end{array}$ & $\begin{array}{l}\text { Tamanho: } 47 \\
\text { veteranos do Vietnã e } \\
50 \text { das OIF/OEF. } \\
\text { Idade: } 60 \pm 2 \\
\text { (Vietnã) e } 30 \pm 6 \text { anos } \\
\text { (OIF/OEF). } \\
\text { Condições clínicas: } \\
\text { perda de membro } \\
\text { superior unilateral. }\end{array}$ & $\begin{array}{l}\text { Foram adaptadas questões da } \\
\text { Houghton Scale, Orthotic and } \\
\text { Prosthetic User's Survey (OPUS) e } \\
\text { Prosthetic Evaluation Questionnaire } \\
\text { (PEQ). As perguntas eram respondidas } \\
\text { por telefone, carta ou pela internet } \\
\text { (REIBER et al., 2010). }\end{array}$ \\
\hline $\begin{array}{l}\text { Kittel, Marco e } \\
\text { Stewart (2002) }\end{array}$ & $\begin{array}{l}\text { Identificar os fatores } \\
\text { que influenciam os } \\
\text { indivíduos com lesão } \\
\text { medular a abandonar a } \\
\text { sua primeira cadeira de } \\
\text { rodas. }\end{array}$ & $\begin{array}{l}\text { Tamanho: três } \\
\text { pessoas. } \\
\text { Idade: maior de } 18 \\
\text { anos. } \\
\text { Condições clínicas: } \\
\text { lesão medular em } \\
\text { nível T7, C7 e T9. }\end{array}$ & $\begin{array}{l}\text { Foram recrutados três indivíduos } \\
\text { exigindo uma cadeira de rodas manual } \\
\text { para a mobilidade independente e que } \\
\text { abandonaram sua primeira cadeira de } \\
\text { rodas. } \\
\text { Foi realizada uma entrevista } \\
\text { semiestruturada de } 45 \text { minutos, } \\
\text { nas casas dos participantes. Cada } \\
\text { entrevista foi audiogravada e as fitas } \\
\text { transcritas na íntegra para fins de } \\
\text { análise de dados. Notas de campo } \\
\text { foram tomadas durante e após a } \\
\text { entrevista. }\end{array}$ \\
\hline $\begin{array}{l}\text { Mukherjee e } \\
\text { Samanta (2005) }\end{array}$ & $\begin{array}{l}\text { Realizar um } \\
\text { levantamento das } \\
\text { dificuldades encontradas } \\
\text { pelos usuários, } \\
\text { identificando: } \\
\text { - a causa da rejeição; } \\
\text { • o destino das cadeiras } \\
\text { de rodas doadas; } \\
\text { - avaliar o desempenho, } \\
\text { analisando a tensão } \\
\text { fisiológica dos } \\
\text { beneficiários durante } \\
\text { a sua deambulação } \\
\text { de rotina usando } \\
\text { parâmetros } \\
\text { cardiorrespiratórios. }\end{array}$ & $\begin{array}{l}\text { Tamanho: } 162 \\
\text { pessoas. } \\
\text { Idade: } 31,6 \pm 8,53 \\
\text { (ocasionais) e } 29,9 \pm \\
6,63 \text { (regulares). } \\
\text { Condições clínicas: } \\
\text { não especificado. }\end{array}$ & $\begin{array}{l}\text { Uma entrevista pessoal foi organizada } \\
\text { com história clínica e destino } \\
\text { das cadeiras de rodas. Para os } \\
\text { participantes que rejeitaram a cadeira } \\
\text { de rodas, eles deveriam escolher um } \\
\text { ponto da lista de respostas como a } \\
\text { causa da rejeição. Um teste de campo } \\
\text { simples foi administrado, no qual } \\
\text { os grupos de participantes foram } \\
\text { instruídos a propulsionar a cadeira de } \\
\text { rodas a uma velocidade sustentada } \\
\text { durante } 5 \text { minutos. A velocidade foi } \\
\text { calculada e a frequência cardíaca foi } \\
\text { monitorada. }\end{array}$ \\
\hline
\end{tabular}


Tabela 1. Continuação...

\begin{tabular}{|c|c|c|c|}
\hline $\begin{array}{l}\text { Autor e ano de } \\
\text { publicação }\end{array}$ & Objetivo & Amostra & Protocolo de coleta de dados \\
\hline Smith et al. (2002) & $\begin{array}{l}\text { Explorar os fatores } \\
\text { que afetam a aceitação } \\
\text { dos dispositivos de } \\
\text { assistência por pessoas } \\
\text { idosas na cidade de } \\
\text { Victoria influenciando o } \\
\text { seu uso. }\end{array}$ & $\begin{array}{l}\text { Tamanho: } 40 \\
\text { pessoas. } \\
\text { Idade: } 65-94 \text { anos. } \\
\text { Condições clínicas: } \\
\text { idosos sem } \\
\text { comprometimento } \\
\text { cognitivo } \\
\text { significativo, } \\
\text { com variedades } \\
\text { de diagnósticos, } \\
\text { incluindo } \\
\text { Acidente Vascular } \\
\text { Encefálico, fraturas, } \\
\text { artrite e doenças } \\
\text { neuromotoras. }\end{array}$ & $\begin{array}{l}\text { Foram selecionados idosos sem } \\
\text { comprometimento cognitivo que } \\
\text { usavam dois ou mais dispositivos de } \\
\text { assistência por pelo menos dois meses. } \\
25 \text { pessoas participaram em um grupo } \\
\text { focal e } 15 \text { pessoas participaram de } \\
\text { entrevistas domiciliares. } \\
\text { Os grupos focais discutiram questões, } \\
\text { como: o uso, a escolha, os métodos } \\
\text { de fornecimento e acompanhamento, } \\
\text { a reciclagem, o empréstimo e o custo } \\
\text { dos dispositivos de assistência. As } \\
\text { sessões foram gravadas, transcritas e } \\
\text { os dados analisados para discutir cada } \\
\text { questão. } \\
\text { As entrevistas domiciliares } \\
\text { exploraram as perguntas dos grupos } \\
\text { focais e o pesquisador observou } \\
\text { todos os dispositivos de assistência } \\
\text { presentes na casa do usuário, que } \\
\text { foi convidado a falar acerca da } \\
\text { aquisição e do uso do dispositivo. As } \\
\text { informações foram resumidas e foram } \\
\text { identificados temas consistentes. }\end{array}$ \\
\hline Fager et al. (2006) & $\begin{array}{l}\text { Documentar a aceitação } \\
\text { da comunicação } \\
\text { aumentativa e alternativa } \\
\text { (CAA), e padrões de } \\
\text { uso de adultos com } \\
\text { traumatismo crânio- } \\
\text { encefálico (TCE) que } \\
\text { usaram os dispositivos } \\
\text { ou estratégias de alta } \\
\text { ou baixa tecnologia, em } \\
\text { algum momento durante } \\
\text { a sua recuperação. }\end{array}$ & $\begin{array}{l}\text { Tamanho: } 25 \\
\text { pessoas. } \\
\text { Idade: } 21-44 \text { anos. } \\
\text { Condições clínicas: } \\
\text { TCE. }\end{array}$ & $\begin{array}{l}\text { Foi aplicado um questionário } \\
\text { com avaliação da CAA em seis } \\
\text { centros regionais especializados } \\
\text { em intervenção para pessoas com } \\
\text { TCE. O questionário incluiu seçães } \\
\text { para documentar (a) informações } \\
\text { gerais e de diagnóstico; (b) o uso do } \\
\text { sistema de CAA; (c) justificativas } \\
\text { detalhadas para a não utilização de } \\
\text { CAA; (d) descrições de formulação } \\
\text { de mensagens e de estratégias de } \\
\text { codificação; (e) o uso de CAA de } \\
\text { baixa tecnologia. }\end{array}$ \\
\hline $\begin{array}{l}\text { Johnson et al. } \\
\text { (2006) }\end{array}$ & $\begin{array}{l}\text { Identificar os fatores que } \\
\text { levaram ao sucesso em } \\
\text { longo prazo dos sistemas } \\
\text { de CAA e quais os } \\
\text { fatores que levaram ao } \\
\text { abandono. }\end{array}$ & $\begin{array}{l}\text { Tamanho: } 275 \\
\text { questionários } \\
\text { devolvidos. } \\
\text { Idade: não apresenta. } \\
\text { Condições clínicas: } \\
\text { não apresenta. }\end{array}$ & $\begin{array}{l}\text { O estudo contou com três fases } \\
\text { previstas: Fase } 1 \text { envolveu o uso } \\
\text { de um grupo focal formado por } \\
\text { fonoaudiólogos, que desenvolveram } \\
\text { uma lista inicial de fatores que } \\
\text { consideravam ser associados com } \\
\text { o sucesso em longo prazo versus } \\
\text { abandono inapropriado de CAA; na } \\
\text { Fase } 2 \text {, as opiniões foram obtidas } \\
\text { a partir de uma amostra maior de } \\
\text { fonoaudiólogos, a fim de confirmar } \\
\text { e completar os fatores identificados } \\
\text { na Fase } 1 \text {; na Fase } 3 \text {, fonoaudiólogos } \\
\text { foram questionados usando essas listas } \\
\text { de fatores, composta por } 106 \text { questões, } \\
\text { com } 41 \text { questões abordando o sucesso } \\
\text { em longo prazo e } 32 \text { abordando o } \\
\text { abandono de AAC. }\end{array}$ \\
\hline
\end{tabular}


Tabela 2. Resultados e fatores relacionados ao abandono.

\begin{tabular}{|c|c|c|}
\hline $\begin{array}{l}\text { Autor e ano de } \\
\text { publicação }\end{array}$ & Resultados & Fatores relacionados ao abandono \\
\hline Verza et al. (2006) & $\begin{array}{l}\text { No total de DTA: } 151 \text { ( } 67 \text { no período } \\
\text { de pré-intervenção e } 84 \text { no período de } \\
\text { intervenção). } \\
\mathbf{N}^{0} \text { de DTAs abandonados: } 25 \text { na pré- } \\
\text { intervenção ( } 37,3 \%) \text { e oito na intervenção } \\
(9,5 \%) \text {. } \\
\text { Tipos de DTA abandonados: colchões } \\
\text { de ar (71\%), órteses de coaptação (100\%), } \\
\text { bengalas ( } 25 \%) \text {, cadeiras de rodas elétricas } \\
(50 \%) \text {, cadeiras de rodas manuais ( } 25 \%) \text {, } \\
\text { estabilizadores (100\%), transferidores } \\
(50 \%) \text { e andadores ( } 75 \%) \text { (na pré- } \\
\text { intervenção); bengalas ( } 50 \%) \text {, órteses de } \\
\text { coaptação ( } 40 \%) \text { e transferidores ( } 50 \%) \text { (na } \\
\text { intervenção). } \\
\text { Tempo de uso antes do abandono: } 13 \\
(52 \%) \text { na pré-intervenção e seis ( } 75 \%) \text { na } \\
\text { intervenção nunca foram usados. Durante } \\
\text { o período de intervenção, oito ( } 24 \%) \text { foram } \\
\text { abandonados depois de dois anos de uso. }\end{array}$ & $\begin{array}{l}\text { - Piora no estado físico; } \\
\text { - Não aceitação do dispositivo; } \\
\text { - Inadequação/Inapropriação do } \\
\text { dispositivo; } \\
\text { - Falta de informação e treinamento. }\end{array}$ \\
\hline $\begin{array}{l}\text { Laferrier et al. } \\
\text { (2010) }\end{array}$ & $\begin{array}{l}\mathbf{N}^{0} \text { total de DTA: } 606 \text { próteses ( } 319 \text { no } \\
\text { grupo Vietnã e } 287 \text { no OIF/OEF) e } 597 \\
\text { cadeiras de rodas ( } 312 \text { no grupo Vietnã e } \\
285 \text { no OIF/OEF). } \\
\mathbf{N}^{0} \text { de DTAs abandonados: para todos os } \\
\text { tipos de próteses, foram abandonados } 54 \\
\text { (17\%) no grupo Vietnã e } 14 \text { ( } 5 \% \text { ) no grupo } \\
\text { OIF/OEF. Para as cadeiras de rodas, foram } \\
\text { abandonadas } 48 \text { ( } 15,4 \% \text { ) no grupo Vietnã e } \\
\text { oito (2,8\%) no grupo OIF/OEF. } \\
\text { Tipos de DTA abandonados: cadeira de } \\
\text { rodas e próteses. } \\
\text { Tempo de uso antes do abandono: } \\
\text { no grupo Vietnã, as próteses foram } \\
\text { abandonadas em um período médio de } 13,9 \\
\text { anos para aqueles com perda de membro } \\
\text { inferior unilateral, em um período médio } \\
\text { de } 6,7 \text { anos para aqueles com perda de } \\
\text { membro inferior bilateral, e em um ano para } \\
\text { aqueles com perda de vários membros. No } \\
\text { grupo OIF/OEF, todas as próteses foram } \\
\text { abandonadas em um ano. }\end{array}$ & $\begin{array}{l}\text { Abandono da cadeira de rodas: } \\
\text { - Peso elevado; } \\
\text { - Comorbidades; } \\
\text { - Condições vasculares; } \\
\text { - Insatisfação com o próprio dispositivo. } \\
\text { Abandono das próteses: } \\
\text { - Preferência pelo uso da cadeira de } \\
\text { rodas; } \\
\text { - Dor; } \\
\text { - Nível da amputação. }\end{array}$ \\
\hline
\end{tabular}


Tabela 2. Continuação...

\begin{tabular}{|c|c|c|}
\hline $\begin{array}{l}\text { Autor e ano de } \\
\text { publicação }\end{array}$ & Resultados & Fatores relacionados ao abandono \\
\hline $\begin{array}{l}\text { Gailey et al. } \\
(2010)\end{array}$ & $\begin{array}{l}\mathbf{N}^{0} \text { total de DTA: } 1738 \text { no grupo Vietnã e } \\
1167 \text { no grupo OIF/OEF. } \\
\text { No }^{\circ} \text { de pessoas que abandonaram os } \\
\text { DTAs: } 19 \text { (11\%) no grupo Vietnã e sete } \\
\text { (4\%) no grupo OIF/OEF. } \\
\text { Tipos de DTA abandonados: próteses } \\
\text { de membro inferior classificadas como: } \\
\text { tecnologia avançada (híbrida, mioelétrica } \\
\text { ou tipo microprocessador), mecânica, } \\
\text { especializada (atléticas, recreativa ou de alto } \\
\text { impacto), à prova d'água e cosmético (não } \\
\text { funcional). } \\
\text { Tempo de uso antes do abandono: as } \\
\text { próteses mecânicas foram utilizadas em uma } \\
\text { média de } 13 \pm 13,5 \text { anos antes de abandono } \\
\text { (variando de seis meses a } 36 \text { anos). Não foi } \\
\text { informado sobre os outros tipos. }\end{array}$ & $\begin{array}{l}\text { - Comprimento residual de membros } \\
\text { insuficiente para suportar o peso } \\
\text { corporal; } \\
\text { - Peso elevado; } \\
\text { - Dor; } \\
\text { - Problemas com a perna contralateral } \\
\text { (traumas cumulativos); } \\
\text { - Ferimentos de combate. } \\
\text { Razões para a rejeição: } \\
\text { - Insatisfação; } \\
\text { - "Muito barulho"; } \\
\text { - Peso elevado; } \\
\text { - Necessidade de recarregar as baterias; } \\
\text { - Dor; } \\
\text { - "Cresceu fora dele"; } \\
\text { - Pobre ajuste. }\end{array}$ \\
\hline $\begin{array}{l}\text { McFarland et al. } \\
(2010)\end{array}$ & $\begin{array}{l}\mathbf{N}^{0} \text { total de DTA: } 250 \text { no grupo Vietnã e } \\
228 \text { no grupo OIF/OEF. } \\
\mathbf{N}^{0} \text { de pessoas que abandonaram os } \\
\text { DTAs: } 14 / 47(30 \%) \text { do grupo Vietnã e } 11 / 49 \\
\text { (22\%) do grupo OIF/OEF abandonaram } \\
\text { todos os dispositivos de prótese para a sua } \\
\text { extremidade superior. } \\
\text { Tipos de DTA abandonados: cadeira de } \\
\text { rodas e próteses. } \\
\text { Tempo de uso antes do abandono: média } \\
\text { de } 4 \pm 8,2 \text { anos (intervalo }=2 \text { meses a } 30 \\
\text { anos de uso no grupo Vietnã, e média de } 8 \\
\pm 7 \text { meses (intervalo }=1 \text { mês a } 1 \text { ano de uso) } \\
\text { no grupo OIF/OEF. }\end{array}$ & $\begin{array}{l}\text { - "Muito barulho"; } \\
\text { - Dor; } \\
\text { - Peso elevado; } \\
\text { - Cotos curtos (não poderia apoiar o } \\
\text { dispositivo); } \\
\text { - Preferência e necessidades por usar } \\
\text { membros residuais; } \\
\text { - Incapacidade de controlar o dispositivo. } \\
\text { Razões para a rejeição: } \\
\text { - Dor; } \\
\text { - Desconforto; } \\
\text { - Falta de funcionalidade; }\end{array}$ \\
\hline $\begin{array}{l}\text { Kittel, Marco e } \\
\text { Stewart (2002) }\end{array}$ & $\begin{array}{l}\mathbf{N}^{0} \text { total de DTA: seis. } \\
\mathbf{N}^{0} \text { de DTAs abandonados: no mínimo, três } \\
(50 \%) \text {. } \\
\text { Tipos de DTA abandonados: cadeira de } \\
\text { rodas manual. } \\
\text { Tempo de uso: entre um e cinco anos. }\end{array}$ & $\begin{array}{l}\text { - Falta de experiência no uso e seleção da } \\
\text { cadeira de rodas; } \\
\text { - Limitações funcionais encontradas } \\
\text { com o desenho, a forma e outras } \\
\text { características da cadeira de rodas; } \\
\text { - Insatisfação e frustração. }\end{array}$ \\
\hline $\begin{array}{l}\text { Mukherjee e } \\
\text { Samanta (2005) }\end{array}$ & $\begin{array}{l}\mathbf{N}^{0} \text { total de DTA: } 162 \\
\mathbf{N}^{0} \text { de pessoas que abandonaram os } \\
\text { DTAs: } 116(71,6 \%) \text {. } \\
\text { Tipos de DTA abandonados: cadeira de } \\
\text { rodas manual. } \\
\text { Tempo de uso antes do abandono: não } \\
\text { informado. }\end{array}$ & $\begin{array}{l}\text { - Dor; } \\
\text { - Fadiga; } \\
\text { - Desconforto; } \\
\text { - Envolvimento dos membros superiores; } \\
\text { - Não adaptação domiciliar; } \\
\text { - Danos frequentes; } \\
\text { - Incapacidade de tocar a cadeira de } \\
\text { rodas. }\end{array}$ \\
\hline Smith et al. (2002) & $\begin{array}{l}\mathbf{N}^{\mathbf{0}} \text { de pessoas que abandonaram os DTAs: } \\
14 \text { interromperam o uso de pelo menos um } \\
\text { item ( } 93,3 \% \text { dos } 15 \text { entrevistados). } \\
\text { Tipos de DTA abandonados: não havia } \\
\text { nenhum padrão, variando de pequenos } \\
\text { até grandes dispositivos auxiliares de } \\
\text { locomoção (cadeira de rodas) e dispositivos } \\
\text { de casa e banho. } \\
\text { Tempo de uso: entre um e cinco anos. }\end{array}$ & $\begin{array}{l}\text { - Custo elevado; } \\
\text { - Falta de participação na escolha do } \\
\text { equipamento; } \\
\text { - Falta de instrução sobre o uso dos } \\
\text { dispositivos; } \\
\text { - Alteração na condição do equipamento; } \\
\text { - Obtenção de um dispositivo melhor; } \\
\text { - Inadequação/Inapropriação do } \\
\text { dispositivo; } \\
\text { - Vergonha de utilizar o dispositivo. }\end{array}$ \\
\hline
\end{tabular}


Tabela 2. Continuação...

\begin{tabular}{|c|c|c|}
\hline $\begin{array}{l}\text { Autor e ano de } \\
\text { publicação }\end{array}$ & Resultados & Fatores relacionados ao abandono \\
\hline Fager et al. (2006) & $\begin{array}{l}\mathbf{N}^{\mathbf{0}} \text { de pessoas que abandonaram os } \\
\text { DTAs: dois de } 15 \text { abandonaram, um rejeitou } \\
\text { e um não teve condições financeiras para } \\
\text { receber DTA de alta tecnologia }(23,5 \%) \text {; } \\
\text { e três em oito abandonaram DTA de baixa } \\
\text { tecnologia }(37,5 \%) \text {. } \\
\text { Tipos de DTA abandonados: CAA de alta }\end{array}$ & $\begin{array}{l}\text { - Falta de apoio facilitador em curso; } \\
\text { - Recuperação da fala natural funcional; } \\
\text { - Limitações físicas graves (representam } \\
\text { uma barreira para o uso do CAA de alta } \\
\text { tecnologia); } \\
\text { - Opção de usar a voz natural. }\end{array}$ \\
\hline
\end{tabular}

e baixa tecnologia.

Tempo de uso: período prolongado (não especificado).

Johnson et al. (2006)
$\mathbf{N}^{\mathbf{0}}$ de pessoas que usaram DTA: $\mathrm{M}=96,89$ $(\mathrm{SD}=132,32)$.

$\mathbf{N}^{0}$ de pessoas que abandonaram os

DTAs: $\mathrm{M}=26,59(\mathrm{SD}=71,95)$.

Tipos de DTA abandonados: CAA

Tempo de uso: um ano.
- Profissionais acreditam que podem compreender pessoa sem necessidade de CAA;

- Falta de oportunidades e motivação por parte dos profissionais;

- Preferência por usar meios de comunicação mais simples;

- Profissionais que trabalham com o usuário CAA não são treinados para operar e/ou programar o sistema;

- Vocabulários/mensagens não atendem às necessidades individuais de vida diária;

- Falta de tempo dos profissionais para treinamento, acompanhamento, colaboração em equipe;

- Falta de apoio de membros da família;

- Usuário é independente, sem necessidade de comunicação com ajuda;

- Vocabulários/Mensagens não estão servindo a uma variedade de funções comunicativas (por exemplo, pedidos, protestos, comentários, narrativas, piadas);

- Sistema difícil de usar;

- A família não é treinada para operar e/ ou programar o sistema;

- A falta de motivação por parte do utilizador;

- Pouco tempo para o sistema manter-se;

- Pouco tempo para a formação de acompanhamento de usuário e parceiros;

- Pouco tempo para a colaboração com os novos membros da equipe em novos locais;

- Não manter ou ajustar o sistema. um, devido à opção de usar o discurso natural, e outro, devido ao custo elevado. Em outro artigo, de Verza et al. (2006), seis pessoas rejeitaram devido à não aceitação do diagnóstico de esclerose múltipla.

A forma de coleta de dados que predominou nos estudos encontrados foi a entrevista. As entrevistas semiestruturadas, utilizadas por três artigos, favorecem as respostas mais claras e voltadas para o objetivo, pois permitem ao informante a possibilidade de discorrer sobre suas experiências, a partir do foco principal proposto e da direção feita pelo entrevistador, enquanto que a entrevista estruturada, utilizada em seis artigos, pode limitar e enrijecer as respostas (BONI; QUARESMA, 2005). Além destas, 
também se verificaram nos artigos entrevistas com grupos focais, os quais, segundo Boni e Quaresma (2005), estimulam a discussão de um tema comum, como um debate aberto. Entretanto, o grupo focal apresenta limitaçóes quanto à dificuldade em explorar questôes em detalhes e ao fato de o ponto de vista de alguns participantes ser dominante sobre os demais (SMITH et al., 2002).

Considerando-se os resultados referentes aos fatores de abandono (Figura 2), verifica-se que problemas com o estado físico do usuário são os fatores de abandono mais citados nos estudos. No estudo de Verza et al. (2006), esse item está ligado à piora do estado físico que acontece com as pessoas com esclerose múltipla. Doenças progressivas tendem a limitar as habilidades funcionais com o tempo, gerando as necessidades de um processo contínuo de reavaliaçóes e reajustes dos DTAs (PAPE; KIM; WEINER, 2002). Verza et al. (2006) ainda concluem que a equipe de saúde deve acompanhar os primeiros três anos após a obtenção do DTA, por este ter sido considerado o "período de risco", tendo em vista que todos os DTAs foram abandonados antes deste período. Três estudos relacionados a amputaçóes de membros superiores e inferiores mencionam os cotos curtos como um fator para o abandono de próteses (KITTEL; MARCO; STEWART, 2002; SMITH et al., 2002; JOHNSON et al., 2006). Ramos e Salles (2003) relatam que, em cotos muito curtos, os movimentos remanescentes utilizados para o comando dos componentes da prótese poderão ser dificultados, prejudicando a correta utilização do DTA. A prótese estética aparece como uma opçáo muito utilizada nesses casos; entretanto, nem todos os amputados são candidatos à protetização, se o indivíduo realizar de forma independente suas atividades. Devem-se analisar a habilidade, a necessidade e os objetivos da pessoa; por isso, a opção pelo não uso do DTA, diante desse cenário, nâo precisa ser encarado como uma falha do processo de reabilitação (RAMOS; SALLES, 2003).

O segundo item mais citado nos estudos é a falta de informação ou treinamento, tanto do usuário como do profissional responsável pela prescrição. Ao adquirir DTAs pré-fabricados, os usuários podem não ter acesso a orientaçóes básicas para a compra e nem a intervenção profissional para o treinamento (PHILLIPS; ZHAO, 1993). Segundo Scherer (1996), pela fácil obtenção comercial do DTA - como a compra em farmácias -, por náo serem dadas as informaçóes adequadas, é grande a chance de abandono. Assim, o usuário pode ter dificuldades para comprar o DTA mais apropriado e, por ser comercial, pode náo atender a suas demandas individuais e específicas, levando ao abandono. $\mathrm{O}$ estudo de Johnson et al. (2006) aponta a falta de informação e treinamento dos próprios profissionais como fator de abandono. No estudo de Verza et al. (2006), por outro lado, verificou-se que uma equipe interdisciplinar é capaz de diminuir as taxas de abandono e relata-se que, durante o período de intervenção, nenhum dispositivo foi abandonado devido à inadequação ou devido à falta de informação. Esses resultados indicam, portanto, a importância do profissional capacitado no processo de prescrição dos DTAs.

Outro fator de abandono citado com frequência foi a dor, uma sensação desagradável que pode ser sinal de lesão corporal. A dor acompanha quase todos os processos de deterioraçáo da saúde e envolve o bem-estar, o sono, o social, o emocional, as habilidades físicas, entre outros aspectos, dificultando o desempenho ocupacional do indivíduo e sua participação (NUNES, 2007). Esse resultado indica a necessidade de o DTA ser ajustado para não propiciar dor, para evitar as lesôes, seja diminuindo o peso do dispositivo ou criando um design mais confortável, e em alguns casos, métodos para diminuir a dor podem ser utilizados antes de começar a utilização dos DTAs (LAFERRIER et al., 2010).

A falta de funcionalidade do DTA foi um item bastante citado como fator de abandono. No estudo de Johnson et al. (2006), os vocabulários/mensagens dos sistemas de comunicação alternativa e aumentada (CAA) não atendem às funçóes comunicativas, e, no estudo de Kittel, Marco e Stewart (2002), são encontradas limitações em relação ao desenho, à forma e a outras características da cadeira de rodas, gerando falta de funcionalidade. Os DTAs têm objetivo de manter ou melhorar as capacidades funcionais das pessoas com deficiência; entáo, se estes estão causando limitações funcionais, não estão cumprindo seu papel. Isso justifica a preferência pelo uso de suas capacidades remanescentes ou de outros DTAs que realmente atendem a suas necessidades.

Embora a inadequação/inapropriação tenha sido apontada como um fator de abandono em dois artigos, os autores não explicitam maiores informaçôes. Acredita-se, porém, que juntamente com o fator de abandono 'insatisfaçáo', apontado por Laferrier et al. (2010), Gailey et al. (2010), Kittel et al. (2002), estejam os fatores relacionados às características dos dispositivos, como peso elevado, design não confortável, limitaçôes na função, mobilidade e acesso, desconforto físico e dificuldades de transporte. Dentre essas características, o peso elevado, o desconforto e "muito barulho" são fatores de abandono específicos citados em vários estudos (LAFERRIER et al., 2010; GAILEY et al., 2010; 
MCFARLAND et al., 2010; MUKHERJEE; SAMANTA, 2005), sempre relacionados à cadeira de rodas e próteses. O desconforto causado pelo dispositivo é apontando por Mcfarland et al. (2010) como um fator de abandono, visto que muitas vezes leva a dor e insatisfação, como o desconforto nos membros superiores ao propulsionar cadeira de rodas devido à força muscular insuficiente, rigidez na parte superior do corpo e baixos níveis de aptidão física (capacidade cardiorrespiratória).

As alteraçôes das condiçóes do DTA também foram consideradas um fator do abandono, pois o seu bom desempenho é essencial à satisfação do cliente, que espera obter com esse dispositivo uma melhoria de sua condição inicial (PHILLIPS; ZHAO, 1993). Sobre isso, os estudos abordam: deterioraçáo dos equipamentos (SMITH et al., 2002), falta de ajustamentos contínuos (JOHNSON et al., 2006) e danos frequentes (como danos de rodas, rolamentos e quebras de eixo em cadeiras de rodas) (MUKHERJEE; SAMANTA, 2005). Esses fatores alteram efetividade, operabilidade e durabilidade dos dispositivos. Percebe-se que a qualidade e o desempenho dos dispositivos são essenciais para que o indivíduo sinta-se motivado a usar o DTA prescrito (PHILLIPS; ZHAO, 1993).

Outro fator abordado para o abandono de DTA é a dificuldade de uso, seja por característica do dispositivo ou do usuário. Os estudos trazem, como exemplo: dificuldade em usar sistemas de CAA, incapacidade de controlar o dispositivo, incapacidade de tocar a cadeira de rodas devido às demandas fisiológicas elevadas para a propulsão e mecanismos biomecânicos ineficientes. Assim, é necessário um envolvimento por parte do fabricante no sentido de criar sistemas mais acessíveis, fornecendo maiores instruçôes. Além disso, é crucial que tais recursos sejam intermediados sempre por algum especialista, que procure favorecer o alcance dos objetivos que visem a igualar e qualificar as oportunidades de interação e comunicação do indivíduo, a educação e as possibilidades de lazer, de profissionalização e de controle e manejo do próprio ambiente, fornecendo assim maior autonomia (HOGETOP; SANTAROSA, 2002).

Outros fatores subjacentes ao abandono foram: falta de participação na escolha do equipamento, falta de motivação por parte do utilizador e vergonha de utilizar o dispositivo. A TA, muitas vezes, é considerada um símbolo da incapacidade que, embora promovido como um meio para aumentar a autonomia funcional, o dispositivo pode ser visto pelo indivíduo como uma validação de estar doente, perder a independência e uma autoimagem diminuída
(VERZA et al., 2006). Aqueles indivíduos que estão desmotivados, intimidados pela tecnologia, envergonhados pela sua utilização, impacientes ou impulsivos, ou que possuem baixa autoestima e expectativas não realistas, além de limitação das habilidades necessárias para sua utilização, podem se tornar usuários parciais ou até mesmo relutantes da TA. Assim, o uso bem sucedido da TA ocorre quando o usuário tem motivação para usar o equipamento, além de cooperação, otimismo e habilidade na sua utilização (COOK; HUSSEY, 2002). Para isso, Kittel et al. (2002) afirmam que a participação ativa do usuário, somada a uma abordagem interdisciplinar na prescrição e na seleção de DTA, é imprescindível para aumentar a adesão e promover resultados efetivos.

\section{Considerações finais}

Este estudo constatou que os fatores mais citados relacionados ao abandono dos dispositivos são: problemas com o estado físico do usuário; falta de informaçáo e treinamento tanto de profissionais quanto de usuários; dor; limitaçóes funcionais; preferência por outro DTA ou utilização de capacidades remanescentes; peso elevado do dispositivo; alteraçóes nas condiçóes do DTA; dificuldade de uso; insatisfação; desconforto; inadequação/inapropriaçấo, e "muito barulho". Compreender melhor os fatores de abandono é fundamental para a prescrição de um DTA e a melhora da eficácia da intervenção, com foco na qualidade de vida dos indivíduos.

A partir deste estudo, indicamos as seguintes sugestóes para promover resultados efetivos e diminuir o índice de abandono dos dispositivos: o profissional deve analisar as habilidades, as necessidades, o contexto e os objetivos da pessoa; abordagem interdisciplinar na prescrição e na seleção de DTA; participação ativa do usuário na prescrição e na seleção de DTA; atenção ao confeccionar o DTA para não propiciar dor, desconforto, insatisfação, entre outros; acompanhamento profissional, para treinamento e orientações específicas; reavaliações e reajustes dos DTAs, e envolvimento por parte do fabricante no sentido de criar sistemas mais acessíveis e adequados ao usuário.

Muitos fatores de abandono descritos estáo interligados. Insatisfação, inadequação e dificuldade de uso são termos amplos, que podem estar associados ao peso elevado do DTA, à dor e ao desconforto, por exemplos. Sugere-se, para estudos futuros, buscar razóes mais detalhadas sobre o abandono, para ajudar no processo de seleção, prescrição, fabricação e uso destes dispositivos, procurando garantir a máxima eficácia da indicação do DTA. 
Além disso, recomenda-se pesquisar fatores de abandono relacionados a outras áreas de aplicaçáo da TA, como mencionado por Mello (1999), pois os artigos desta revisão abordam as áreas de órteses e próteses, mobilidade e comunicação alternativa.

\section{Referências}

ANDRADE, V. S.; PEREIRA, L. S. M. Influência da tecnologia assistiva no desempenho funcional e na qualidade de vida de idosos comunitários frágeis: uma revisão bibliográfica. Revista Brasileira de Geriatria e Gerontologia, Rio de Janeiro, v. 12, n. 1, p. 113-122, 2009. Disponível em: <http://www.crde-unati.uerj.br/img_tse/ v12n1/pdf/art_9.pdf>. Acesso em: 06 ago. 2013.

BERQUÓ, A. F. Biblioteca Louis Braille do Instituto Benjamin Constant: assegurando ao deficiente visual acesso ao conhecimento. Revista $A C B$, Florianópolis, v. 13, n.1, p. 282-290, 2008.

BONI, V.; QUARESMA, S. J. Aprendendo a entrevistar: como fazer entrevistas em Ciências Sociais. Revista Eletrônica dos Pós-Graduandos em Sociologia Politica da UFSC, Florianópolis, v. 2, n. 1, p. 68-80, 2005. Disponível em: <periodicos.ufsc.br/index.php/emtese/article/ viewFile/18027/16976>. Acesso em: 04 out. 2013.

BRASIL. Secretaria dos Direitos Humanos. Plano Nacional dos Direitos da Pessoa com Deficiência: viver sem limite. Brasília, DF, 2013. Disponível em: <http:// www.pessoacomdeficiencia.gov.br/app/sites/default/ files/arquivos/\%5Bfield_generico_imagens-filefield-description\%5D_0.pdf>. Acesso em: 30 maio 2013.

BRASIL. Secretaria Especial dos Direitos Humanos. Coordenadoria Nacional para Integração da Pessoa Portadora de Deficiência. Ata VII reuniäo do Comitê de Ajudas Técnicas - CAT CORDE / SEDH / PR realizada nos dias 13 e 14 de dezembro de 2007. Brasília, DF, 2007. Disponível em: <http://www.infoesp.net/CAT_Reuniao_VII. pdf>. Acesso em: 07 jun. 2013.

COOK, A. M.; HUSSEY, S. M. The Disabled human user of assistive technologies. In: COOK, A. M.; HUSSEY, S. M. Assistive technologies: principles and practice. St. Louis: Mosby, 2002. p. 54-90.

CRUZ, D. M. C. Papéis ocupacionais e pessoas com deficiências fisicas: independência, tecnologia assistiva e poder aquisitivo. 2012. 229 f. Tese (Doutorado em Educação Especial) - Universidade Federal de São Carlos, São Carlos, 2012.

FAGER, S. et al. Augmentative and alternative communication use and acceptance by adults with traumatic brain injury. Augmentative and Alternative Communication, Londres, v. 22, n. 1, p. 37-47, 2006. http://dx.doi. org/10.1080/07434610500243990.

GAILEY, R. et al. Unilateral lower-limb loss: prosthetic device use and functional outcomes in service members from Vietnam war and OIF/OEF conflicts. Journal of Rehabilitation Research \& Development, Washington, v.
47, n. 4, p. 317-332, 2010. Disponível em: <http:// www.rehab.research.va.gov/jour/10/474/Gailey.html>. Acesso em: 11 maio 2013.

HOGETOP, L.; SANTAROSA, L. M. C. Tecnologias assistivas: viabilizando a acessibilidade ao potencial individual. Informática na Educação: Teoria \& Prática, Porto Alegre, v. 5, n. 2, p.1-19, 2002.

INSTITUTO BRASILEIRO DE GEOGRAFIA E ESTATÍSTICA - IBGE. Censo demográfico 2000: características gerais da população. Resultados da amostra. Rio de Janeiro: IBGE, 2003. Disponível em: < http://www. ibge.gov.br/home/estatistica/populacao/censo2000/ populacao/censo2000_populacao.pdf >. Acesso em: 15 maio 2013.

INSTITUTO BRASILEIRO DE GEOGRAFIA E ESTATÍSTICA - IBGE. Censo demográfico 2010: características gerais da população, religião e pessoas com deficiência. Rio de Janeiro: IBGE, 2012. Disponível em: <ftp://ftp.ibge.gov.br/Censos/Censo_Demografico_2010/Caracteristicas_Gerais_Religiao_Deficiencia/ caracteristicas_religiao_deficiencia.pdf $>$. Acesso em: 15 maio 2013.

JOHNSON, J. M. et al. Perspectives of Speech Language Pathologists Regarding Success versus Abandonment of AAC. Augmentative and Alternative Communication, Londres, v. 22, n. 2, p. 85-99, 2006. http://dx.doi. org/10.1080/07434610500483588.

KITTEL, A.; MARCO, A. D.; STEWART, H. Factors influencing the decision to abandon manual wheelchairs for three individuals with a spinal cord injury. Disability and Rehabilitation, Londres, v. 24, n. 1-3, p. 106-114, 2002. Disponível em: <http://informahealthcare.com/ doi/abs/10.1080/09638280110066785?journalCode $=\mathrm{d}$ re>. Acesso em: 11 maio 2013.

LAFERRIER, J. Z. et al. Wheeled mobility: factors influencing mobility and assistive technology in veterans and servicemembers with major traumatic limb loss from Vietnam war and OIF/OEF conflicts. Journal of Rehabilitation Research \& Development, Washington, v. 47, n.4, p. 349-360, 2010. Disponível em: <http://www. rehab.research.va.gov/jour/10/474/Laferrier.html>. Acesso em: 11 maio 2013.

LAW, M. et al. Formulário de revisão crítica - estudos quantitativos. Tradução Raquel E. Luz, Marisa C. Mancini, Rosana F. Sampaio. Belo Horizonte: Universidade Federal de Minas Gerais, 1998. Disponível em: <http:// www.uff.br/especializacaofisioto/files/2010/04/Formulario-an\%C3\%A1lise-de-estudos-quantitativos.pdf>. Acesso em: 04 nov. 2013.

LI, W.; SELLERS, C. Improving assistive technology economics for people with disabilities: harnessing the voluntary and education sectors. In: TORONTO INTERNATIONAL CONFERENCE ON SCIENCE AND TECHNOLOGY FOR HUMANITY (TIC-STH), 2009, Toronto. Proceeding... Toronto: IEEE, 2009. p. 789-794. 
MANCINI, M. C.; SAMPAIO, R. F. Quando o objeto de estudo é a Literatura: estudos de revisão. Revista Brasileira de Fisioterapia, São Carlos, v. 10, n. 4, p. 361-472, 2006.

MCFARLAND, L. V. et al. Unilateral upper-limb loss: satisfaction and prosthetic-device use in veterans and service members from Vietnam and OIF/OEF conflicts. Journal of Rehabilitation Research \& Development, Washington, v. 47, n. 4, p. 299-316, 2010. Disponível em: <http://www.rehab.research.va.gov/jour/10/474/McFarland.html>. Acesso em: 11 maio 2013.

MELLO, M. A. F. Tecnologia Assistiva. In: GREVE, J. M. A.; AMATUZZI, M. M. Medicina de reabilitação aplicada à ortopedia e traumatologia. São Paulo: Roca, 1999. p. 407-419.

MUKHERJEE, G.; SAMANTA, A. Wheelchair charity: a useless benevolence in community-based Rehabilitation. Disability and Rehabilitation, Londres, v. 27, n. 10, p. 591-596, 2005. Disponível em: <http://informahealthcare.com/doi/abs/10.1080/09638280400018387>. Acesso em: 11 maio 2013.

NUNES, C. M. P. Dor neuromusculoesquelética. In: CAVALCANTI, A.; GALVĀO, C. Terapia ocupacional: fundamentação \& prática. Rio de Janeiro: Guanabara Koogan, 2007. p. 258-268.

OLIVEIRA, A. I. A.; GAROTTI, M. F.; SÁ, N. M. C. M. Tecnologia de ensino e Tecnologia Assistiva no ensino de crianças com paralisia cerebral. Ciências \& Cognição, Rio de Janeiro, v. 13, n. 3, p. 243-262, 2008. Disponível em: <http://www.cienciasecognicao.org/revista/index. php/cec/article/view/78>. Acesso em: 06 ago. 2013.

ORGANIZAÇÃO MUNDIAL DA SAÚDE - OMS. Classificação internacional de funcionalidade, incapacidade e saúde - CIF. São Paulo: EDUSP; 2003.

ORGANIZAÇÃO MUNDIAL DA SAÚDE - OMS. Relatório mundial sobre a deficiência. Tradução Lexicus Serviços Linguísticos. São Paulo: Secretaria dos Direitos da Pessoa com Deficiência, 2012.

PAPE, T. L.; KIM, J.; WEINER, B. The shaping of individual meanings assigned to assistive technology: a review of personal factors. Disability and Rehabilitation, Londres, v. 24, n. 1-3, p. 5-20, 2002. Disponível em: <http://www.researchgate.net/publication/11533525_ The_shaping_of_individual_meanings_assigned_to_as- sistive_technology_a_review_of_personal_factors $>$. Acesso em: 11 maio 2013.

PHILLIPS, B.; ZHAO, H. Predictors of assistive technology abandonment. Assistive Technology: The Official Journal of RESNA, Nova Iorque, v. 5, n. 1, p. 36-45, 1993. http://dx.doi.org/10.1080/10400435.1993.1013 2205. PMid:10171664.

RAMOS, A. C.; SALLES, I. C. Amputaçóes de membros superiores. In: TEIXEIRA, E. Terapia ocupacional na reabilitação física. São Paulo: Roca, 2003. p. 17-28.

REIBER, G. E. et al. Servicemembers and veterans with major traumatic limb loss from Vietnam war and OIF/ OEF conflicts: survey methods, participants, and summary findings. Journal of Rehabilitation Research \& Development, Washington, v. 47, n. 4, p. 275-298, 2010. Disponível em: <http://www.rehab.research.va.gov/ jour/10/474/Reiber.html>. Acesso em: 11 maio 2013.

RIEMER-REISS, M. L.; WACKER, R. R. Factors associated with assistive technology discontinuance among individuals with disabilities. Journal of Rehabilitation, Washington, v. 66, n. 3, p. 44-50, 2000.

SCHERER, M. J. Outcomes of assistive technology use on quality of life. Disability and Rehabilitation, Londres, v. 18, n. 9, p. 439-448, 1996. Disponível em: <http:// informahealthcare.com/doi/abs/10.3109/09638289609 165907? journalCode=dre $>$. Acesso em: 11 maio 2013.

SMITH, R. et al. Assistive devices: self-reported use by older people in Victoria. Australian Health Review, Sidney, v. 25, n. 4, p. 169-177, 2002. Disponível em: <http:// www.publish.csiro.au/index.cfm? paper $=$ AH020169>. Acesso em: 11 maio 2013.

UNITES STATES. Public Law 105-394. Assistive Technology Act of 1998. To support programs of grants to States to address the assistive technology needs of individuals with disabilities, and for other purposes. Government Printing Office, Washington, 13 nov. 1998. Disponível em: <http://www.gpo.gov/fdsys/pkg/PLAW-105publ394/pdf/PLAW-105publ394.pdf>. Acesso em: 4 out. 2013.

VERZA, R. et al. An interdisciplinary approach to evaluating the need for assistive technology reduces equipment abandonment. Multiple Sclerosis Journal, Londres, v. 12, n. 1, p. 88-93, 2006. Disponível em: <http://msj. sagepub.com/content/12/1/88>. Acesso em: 11 maio 2013.

\section{Contribuição dos Autores}

Celso Rodrigues da Costa e Fernanda M. Rodrigues M. Ferreira: concepção do texto, coleta de dados, organização, análise e interpretação dos resultados, revisão do texto. Marcos Vinícius Bortolus: revisão do texto. Maria Gabriela Reis Carvalho: orientação da pesquisa, interpretação dos resultados, revisão do texto. Todos os autores aprovaram a versão final do texto.

\section{Notas}

${ }^{1}$ Resultado do Trabalho de Conclusão de Curso da Graduação em Terapia Ocupacional na Universidade Federal de Minas Gerais, em novembro de 2013. 